195

\section{Current Use and Trends in the Management of} Tardive Dyskinesia: Role of VMAT2 Inhibitors

Venkatesh Sreeram, $M B B S^{\prime}$; Shanila Shagufta, MD, $\mathrm{MPH}^{2}$; Faisal Kagadkar, MBBS ; and Mustafa Qureshi, $M D^{4}$

${ }^{1}$ Psychiatry, Northwell Health- Zucker Hillside

Hospital, New York, NY

${ }^{2}$ Behavior Health Sciences, New York Medical College, New York, NY

${ }^{3}$ Psychiatry, Kings County Hospital Center, New York City, NY

${ }^{4}$ Psychiatry, Texas Tech University Health Sciences

Center, Permian Basin, TX

ABSTRACT: Objectives: Tardive Dyskinesia (TD) is a debilitating condition that requires prompt care and intervention. Studies demonstrated the probable role of Vesicular Monoamine Transport 2 (VMAT2) in the pathogenesis of TD and use of VMAT2inhibitors in managing TD. Our aim is to provide available data on the management of TD and to determine the efficacy ofvarious VMAT2 inhibitors for independent use. Also, to identify their use in combination and assess if there, any change inoutcome with early intervention.

METHODS: We did a pivotal search of the scientific literature by querying PubMed and Google Scholar for studies on treatment modalities of TD including atypical antipsychotics and VMAT2 inhibitors. Also, references from publications were accessed for review.

RESULTS: Early detection and prevention are of paramount importance in managing TD. Cessate the antipsychotic been using andother dopamine blocking agents that probably implicated in causing the symptoms. Certain studies showed the use of new atypical antipsychotics like Paliperidone, Quetiapine in small titrated dose resolving the symptoms. According to the available data, using benzodiazepines, botulinum toxin injections and VMAT2 inhibitors like Valbenazine, Tetrabenazine, andDeutetrabenazine also managed TD with efficacy. Valbenazine has breakthrough global approval in resolving the symptoms. Although other VMAT2 inhibitors were used for TD earlier and showed to be effective in managing TD, larger trials are required showing their safety and reliability in efficacy.

CONCLUSIONS: VMAT2 inhibitors were tested as efficacious in managing TD. Valbenazine and recently deutetrabenazine has been approved by US Food and Drug Administration (FDA) to treat TD. However, Tetrabenazine is yet to be approved by FDA. More clinical trials are required exploring their efficacy by comparing them or using them in combination. Our review also suggests timely detection and earlier intervening, especially if witnessed in children and adolescents would differ the outcome of TD.

FUNDING ACKNOWLEDGEMENTS: No funding.

197

\section{Guanfacine and Impulsivity - Review of Literature}

William Donovan Vargas' ${ }^{\text {; }}$ and Nevena V. Radonjü'

${ }^{1}$ Department of Psychiatry, University of Connecticut, Farmington, USA

ABSTRACT: Prefrontal cortex (PFC) represents one of the most evolved regions of primate brain that is thought to regulate human specific features such as cognition, emotion and behavior (Arnsten and Jin, 2012). PFC is a site of action of guanfacine, an agonist of alpha 2 adrenergic receptors. Compared to clonidine, another alpha adrenergic drug, guanfacine is more selective for 2 A adrenergic receptor subtype (van Zwieten et al., 1994; Uhlen at al., 1995) and is weaker in producing hypotension andsedation (Jurado at al., 1998) resulting in better tolerability of the medication. Studies have shown that endogenous noradrenergic stimulation of alpha2A receptors is essential for PFC regulation of behavior, thought and emotion as blockade of $\alpha 2 \mathrm{~A}$ receptors in the monkey dorsolateral PFC significantly impairs working memory ( $\mathrm{Li}$ and Mei, 1994) and behavioral inhibition (Ma et al., 2003; Ma et al., 2003). So far FDA has approved guanfacine in treatment of attention deficit hyperactivity disorder in children but the medication is used off label for treatment of oppositional defiant disorder, conduct disorder, pervasive developmental disorders, motor tics and Tourette's syndrome as well. Impulsivity as used in clinical terms is very broadly defined and encompasses personality traits as well as cognitive functions such as emotion regulation and behavioral inhibition. Numerous studies have shown effectiveness of extended release guanfacine in reducing impulsiveness in children with ADHD and recently in autism spectrum disorder (Scahill et al., 2015), however limited data is available on use of guanfacine in treatment of impulse control and aggression in adults.

FUNDING ACKNOWLEDGEMENTS: No funding.

198

Who Am I: Delusion of Misidentification of Self

Yara W. Moustafa, MD, $P h D^{\prime}$; and Alican Dalkilic, $M D, M P H^{2,3}$

${ }^{1}$ Resident, Department of Psychiatry, St. Elizabeths Hopsital, DBH, Washington, DC 
${ }^{2}$ Clinical Professor, Department of Psychiatry, Cooper

Medical School of Rowan University, Camden, NJ

${ }^{3}$ Clinical Faculty, St. Elizabeths Hospital, DBH,

Washington, DC

ABSTRACT: Delusional misidentification syndromes are rare psychotic disorders, in which the patient believes that the identity of a person, object, or place has been changed or replaced by another. These disorders include Capgras syndrome, Fregoli syndrome, and intermetamorphosis. Reverse types of misidentification syndromes were introduced to distinguish alteration of the self rather than of others.

Reverse Capgras syndrome refers to the psychological change of the self as opposed to others, while reverse Fregoli syndrome is the delusional belief in which the patient has undergone fundamental changes in physical makeup without any psychological changes, and reverse intermetamorphosis is a variant in which patients believe that they have undergone physical and psychological transformation.

Here we present an interesting case of a transgender woman presenting with delusional misidentification of the self in the context of Schizophrenia. A review of literature, with emphasis on etiological factors, forensic implications and association with violence is presented.

FUNDING ACKNOWLEDGEMENTS: No funding.

199

\section{Cardiac Safety and QTc Intervals With DM/Q Treatment for Pseudobulbar Affect: A Review of Clinical Trial Data}

Jay W. Mason, MD'; Emmanuelle Hugentobler, $\mathrm{MD}^{2}$; Andrea E. Formella, PharmD ${ }^{2}$; and Laura E. Pope, PhD

${ }^{1}$ Mason Cardiac Safety Consulting, Reno, NV

${ }^{2}$ Avanir Pharmaceuticals, Inc., Aliso Viejo, CA

ABSTRACT: OBJECTIVE: Dextromethorphan hydrobromide and quinidine sulfate (DM/Q) $20 \mathrm{mg} / 10 \mathrm{mg}$ is FDA approved to treat pseudobulbar affect (PBA), a neurological condition characterized by sudden, frequent, involuntary crying or laughing. Although the total dose of quinidine $(20 \mathrm{mg})$ from twice daily DM/Q for PBA is well below the antiarrhythmic dose (600-1600 mg/day), clinicians may be reticent to use DM/Q due to concerns for cardiac safety.
METHOD: DM/Q cardiac safety was evaluated in two thorough QTc (TQT) studies and by ECG monitoring in DM/Q phase 3 clinical trials. In the TQT studies, twice daily DM/Q 30mg/10mg (Study 08-AVR-126; N=50 enrolled) and supratherapeutic doses $30 \mathrm{mg} / 30 \mathrm{mg}$ and $60 \mathrm{mg} / 60 \mathrm{mg}$ (Study 05-AVR-119; N = 36 enrolled) were studied in healthy volunteers. In phase 3 controlled clinical trials, the effects of DM/Q on Fridericia's corrected QT intervals $(\mathrm{QTcF})$ were assessed, as was the incidence of ECG outliers. Adverse events (AEs) were monitored in all clinical trials.

RESULTS: Overall, 47/50 participants completed TQT study 08-AVR-126 and 36/36 completed TQT study 05-AVR-119. Time-matched, placebo-corrected mean maximal changes in QTcF for DM/Q $30 \mathrm{mg} / 10 \mathrm{mg}$ and $30 \mathrm{mg} / 30 \mathrm{mg}$ occurred $3 \mathrm{~h}$ post-dose ( 10.3 and $10.1 \mathrm{~ms}$, respectively) vs moxifloxacin $(12.2$ and $14.4 \mathrm{~ms}$ at 1.5 and $1.0 \mathrm{~h})$. For the supratherapeutic DM/Q 60/60 mg dose, mean maximal QTcF change was $18.8 \mathrm{~ms}$. No participant had a QTcF $>480$ ms or QTc increase $>60 \mathrm{~ms}$. In PBA phase 3 controlled trials, mean changes in QTcF were similar for DM/Q containing Q $10 \mathrm{mg}$ (0.4 to $3.5 \mathrm{~ms} ; \mathrm{n}=217)$ andplacebo (0.4 to $3.1 \mathrm{~ms} ; \mathrm{n}=183$ ), but greater for $\mathrm{DM} / \mathrm{Q}$ with $\mathrm{Q} 30$ $\mathrm{mg}$ (2.9 to $7.6 \mathrm{~ms} ; \mathrm{n}=146)$. In an outlier analysis, a similar percentage of DM/Q-treated participants $(3.9 \%)$ had a QTcF shift from $<450 \mathrm{~ms}$ at baseline to $\geq 450 \mathrm{~ms}$ during treatment vs placebo $(2.9 \%)$. No participant with PBA had a QTc change from baseline $>60 \mathrm{~ms}$ or an absolute QTc interval $>480 \mathrm{~ms}$. No dose- or time-related trends in cardiac arrhythmias or other cardiac-related AEs were observed. Of $2552 \mathrm{DM} / \mathrm{Q}$-treated patients and healthy participants across all controlled and open-label trials for any indication, 11 deaths due to any cardiovascular $\mathrm{AE}$ have been reported; none were attributable to DM/Q treatment and none occurred in placebo-controlled PBA clinical trials in any treatment group. Overall 16 patients had a QTcF that exceeded $500 \mathrm{~ms}$ at any ECG measurement.

CONCLUSIONS: The TQT studies demonstrated that DM/Q has the potential to prolong the QT interval in a dosedependent manner, but that the risk for QTc prolongation and arrhythmias with Q $10 \mathrm{mg}$ formulations is low. In clinical trials with PBA patients, the cardiac safety profile of $\mathrm{DM} / \mathrm{Q} 20 \mathrm{mg} / 10 \mathrm{mg}$ or $30 / 10 \mathrm{mg}$ was indistinguishable from placebo.

FUNDING ACKNOWLEDGEMENTS: Avanir Pharmaceuticals, Inc. 